\title{
SN 2017ens: The Metamorphosis of a Luminous Broadlined Type Ic Supernova into an SN IIn
}

T.-W. Chen ${ }^{1}$ (D), C. Inserra ${ }^{2}$ (D) M. Mraser ${ }^{3}$ (D) T. J. Moriya ${ }^{4}$ (D) P. Schady ${ }^{1}$, T. Schweyer ${ }^{1}$, A. V. Filippenko ${ }^{5,6}$ (D), D. A. Perley ${ }^{7}$ (D), A. J. Ruiter ${ }^{8,9}$ (D) I. Seitenzahl ${ }^{8,9}$ (D) , J. Sollerman ${ }^{10}$ (D), F. Taddia ${ }^{10}$, J. P. Anderson ${ }^{11}$ (D), R. J. Foley ${ }^{12}$, A. Jerkstrand ${ }^{13}$ (iD), C.-C. Ngeow ${ }^{14}$ (D) Y.-C. Pan ${ }^{12}$, A. Pastorello ${ }^{15}$, S. Points ${ }^{16}$, S. J. Smartt ${ }^{17}$ (D) K. W. Smith ${ }^{17}$, S. Taubenberger ${ }^{13,18}$ (D), P. Wiseman ${ }^{2}$, D. R. Young $^{17}$, S. Benetti ${ }^{15}$, M. Berton ${ }^{19,20}$ (D), F. Bufano ${ }^{21}$, P. Clark ${ }^{17}$, M. Della Valle ${ }^{22,23,24}$ (D) , L. Galbany ${ }^{25}$ (iD) A. Gal-Yam ${ }^{26}$ (D), M. Gromadzki ${ }^{27}$, C. P. Gutiérrez ${ }^{2}$, A. Heinze ${ }^{28}$ (D) E. Kankare ${ }^{17}$, C. D. Kilpatrick ${ }^{12}$, H. Kuncarayakti ${ }^{19,29}$ (D), G. Leloudas ${ }^{30}$ (D), Z.-Y. Lin $^{14}$, K. Maguire ${ }^{17}$, P. Mazzali ${ }^{7}$ (D) O. McBrien ${ }^{17}$, S. J. Prentice ${ }^{17}$, A. Rau ${ }^{1}$ (D) A. Rest ${ }^{31,32}$, M. R. Siebert ${ }^{12}$, B. Stalder ${ }^{33}$ (D), J. L. Tonry ${ }^{28}$, and P.-C. $\mathrm{Yu}^{14}$ iD

${ }^{1}$ Max-Planck-Institut für Extraterrestrische Physik, Giessenbachstraße 1, D-85748, Garching, Germany; jchen@mpe.mpg.de ${ }^{2}$ Department of Physics and Astronomy, University of Southampton, Southampton SO17 1BJ, UK

${ }^{3}$ School of Physics, O’Brien Centre for Science North, University College Dublin, Belfield, Dublin 4, Ireland

${ }^{4}$ Division of Theoretical Astronomy, National Astronomical Observatory of Japan, National Institutes for Natural Sciences, 2-21-1 Osawa, Mitaka, Tokyo 181-8588, Japan

${ }^{5}$ Department of Astronomy, University of California, Berkeley, CA 94720-3411, USA

${ }^{6}$ Miller Senior Fellow, Miller Institute for Basic Research in Science, University of California, Berkeley, CA 94720, USA

${ }^{7}$ Astrophysics Research Institute, Liverpool John Moores University, IC2, Liverpool Science Park, 146 Brownlow Hill, Liverpool L3 5RF, UK

${ }^{8}$ School of Physical, Environmental and Mathematical Sciences, University of New South Wales, Australian Defence Force Academy, Canberra, ACT 2600, Australia

${ }^{9}$ Research School of Astronomy and Astrophysics, Australian National University, Canberra, ACT 0200, Australia

${ }^{10}$ The Oskar Klein Centre, Department of Astronomy, AlbaNova, Stockholm University, SE-106 91 Stockholm, Sweden ${ }^{11}$ European Southern Observatory, Alonso de Córdova 3107, Casilla 19, Santiago, Chile

12 Department of Astronomy and Astrophysics, University of California, Santa Cruz, CA 95064, USA

${ }^{13}$ Max-Planck Institut für Astrophysik, Karl-Schwarzschild Str. 1, D-85748 Garching, Germany

${ }^{14}$ Graduate Institute of Astronomy, National Central University, No. 300, Zhongda Road, Zhongli District, Taoyuan City 32001, Taiwan

${ }^{15}$ INAF - Osservatorio Astronomico di Padova, Vicolo dell'Osservatorio 5, I-35122 Padova, Italy ${ }^{16}$ CTIO/NOAO, Casilla 603, La Serena, Chile

${ }^{17}$ Astrophysics Research Centre, School of Mathematics and Physics, Queen's University Belfast, Belfast BT7 1NN, UK

${ }_{18}$ European Southern Observatory, Karl-Schwarzschild-Str. 2, D-85748 Garching, Germany

${ }^{19}$ Finnish Centre for Astronomy with ESO (FINCA), FI-20014 University of Turku, Finland

${ }^{20}$ Aalto University Metsähovi Radio Observatory, Metsähovintie 114, FI-02540 Kylmälä, Finland

${ }^{21}$ INAF-Osservatorio Astrofisico di Catania Via Santa Sofia, 78, I-95123, Catania, Italy

${ }^{22}$ INAF-Osservatorio Astronomico di Napoli, Salita Moiariello, 16, I-80131, Napoli, Italy

${ }^{23}$ Instituto de Astrofisica de Andalucia (IAA-CSIC), Glorieta de la Astronomia s/n, E-18008 Granada, Spain

${ }^{24}$ International Center for Relativistic Astrophysics, Piazza della Repubblica 10, I-65122 Pescara, Italy

${ }^{25}$ PITT PACC, Department of Physics and Astronomy, University of Pittsburgh, Pittsburgh, PA 15260, USA

${ }^{26}$ Department of Particle Physics and Astrophysics, Weizmann Institute of Science, Rehovot 76100, Israel

${ }^{27}$ Warsaw University Astronomical Observatory, Al. Ujazdowskie 4, 00-478 Warszawa, Poland

${ }^{28}$ Institute for Astronomy, University of Hawaii, 2680 Woodlawn Drive, Honolulu, HI 96822, USA

${ }^{29}$ Tuorla Observatory, Department of Physics and Astronomy, FI-20014 University of Turku, Finland

${ }^{30}$ DTU Space, National Space Institute, Technical University of Denmark, Elektrovej 327, DK-2800 Kgs. Lyngby, Denmark

${ }^{31}$ Space Telescope Science Institute, 3700 San Martin Drive, Baltimore, MD 21218, USA

${ }^{32}$ Department of Physics and Astronomy, Johns Hopkins University, Baltimore, MD 21218, USA

${ }^{33}$ LSST, 950 North Cherry Avenue, Tucson, AZ 85719, USA

Received 2018 August 12; revised 2018 October 10; accepted 2018 October 20; published 2018 November 9

\begin{abstract}
We present observations of supernova (SN) 2017ens, discovered by the ATLAS survey and identified as a hot blue object through the GREAT program. The redshift $z=0.1086$ implies a peak brightness of $M_{g}=-21.1 \mathrm{mag}$, placing the object within the regime of superluminous supernovae. We observe a dramatic spectral evolution, from initially being blue and featureless, to later developing features similar to those of the broadlined Type Ic SN 1998bw, and finally showing $\sim 2000 \mathrm{~km} \mathrm{~s}^{-1}$ wide $\mathrm{H} \alpha$ and $\mathrm{H} \beta$ emission. Relatively narrow Balmer emission (reminiscent of a SN IIn) is present at all times. We also detect coronal lines, indicative of a dense circumstellar medium. We constrain the progenitor wind velocity to $\sim 50-60 \mathrm{~km} \mathrm{~s}^{-1}$ based on P-Cygni profiles, which is far slower than those present in Wolf-Rayet stars. This may suggest that the progenitor passed through a luminous blue variable phase, or that the wind is instead from a binary companion red supergiant star. At late times we see the $\sim 2000 \mathrm{~km} \mathrm{~s}^{-1}$ wide $\mathrm{H} \alpha$ emission persisting at high luminosity $\left(\sim 3 \times 10^{40} \mathrm{erg} \mathrm{s}^{-1}\right)$ for at least 100 day, perhaps indicative of additional mass loss at high velocities that could have been ejected by a pulsational pair instability.
\end{abstract}

Key words: supernovae: general - supernovae: individual (SN 2017ens)

Supporting material: data behind figure 


\section{Introduction}

Type Ic supernovae (SNe) arise from the core collapse of a massive star that has lost its hydrogen and helium layers prior to exploding, through either strong stellar winds or interaction with a binary companion (e.g., Filippenko 1997; Gal-Yam 2017). Their light curves are powered by the radioactive decay of ${ }^{56} \mathrm{Ni}$ that is produced in the $\mathrm{SN}$ explosion. Related to these events, but with luminosities up to 100 times higher, are the Type I superluminous SNe (SLSNe I; see Gal-Yam 2012; Inserra et al. 2018a; Moriya et al. 2018b for reviews of observations and models). SLSNe exhibit spectral similarities to SNe Ic (Pastorello et al. 2010), but their luminosities are such that they cannot be powered solely by radioactive decay (Quimby et al. 2011). The nature of the additional energy source remains unknown, with suggestions ranging from a central engine (Kasen \& Bildsten 2010; Woosley 2010) to interaction with a massive $\mathrm{H}$ and $\mathrm{He}$-free circumstellar medium (CSM; Chevalier \& Irwin 2011).

Some $\mathrm{SNe} \mathrm{Ib} / \mathrm{Ic}$ have been observed to develop relatively narrow $\left(\sim 500-1000 \mathrm{~km} \mathrm{~s}^{-1}\right)$ emission lines of hydrogen in their spectra; examples include SNe Ib $2014 \mathrm{C}$ and $2004 \mathrm{dk}$ (Milisavljevic et al. 2015; Mauerhan et al. 2018), and SNe Ic 2001em and 2017dio (Gal-Yam 2017; Kuncarayakti et al. 2018). This has been interpreted as evidence that for at least some $\mathrm{H}$-poor $\mathrm{SNe}$, the fast ejecta are colliding with $\mathrm{H}$-rich material relatively far from the star. This late-time interaction has also been observed in some SLSNe Ic which show $\mathrm{H} \alpha$ emission at +70 to +250 day after their peak brightness (Yan et al. 2015, 2017).

In this Letter we report on the discovery of an unusual SN with our Gamma-Ray Burst Optical/Near-Infrared Detector (GROND)/extended-Public ESO Spectroscopic Survey for Transient Objects (ePESSTO)/Asteroid Terrestrial-impact Last Alert System (ATLAS) (GREAT; Greiner et al. 2008; Smartt et al. 2015; Tonry et al. 2018) survey. We introduce this program here, which is designed to rapidly identify hot blue transients, with the specific goal of finding very young SLSNe in faint galaxies (Chen et al. 2017c). SN 2017ens (ATLAS17gqa) was discovered by the ATLAS survey on 2017 June 5 (UT dates are used herein), located at (J2000) $\alpha=12^{\mathrm{h}} 04^{\mathrm{m}} 09^{\mathrm{s}} \cdot 37$, $\delta=-01^{\circ} 55^{\prime} 52$ "' 2 . Prompted by the high blackbody temperature of $21,000 \pm 3000 \mathrm{~K}$ that we measured with our GREAT data on 2017 June 8 (Chen et al. 2017a), we began an intensive spectroscopic and photometric follow-up campaign (Section 2).

The adopted redshift of SN 2017ens, $z=0.1086$ (Section 3.3), implies an absolute magnitude of $M_{g}=-21.1$ at peak, and thus a luminosity consistent with a SLSN (Gal-Yam 2012). In Section 3 we present the spectral evolution of SN 2017ens, which began to show $\sim 2000 \mathrm{~km} \mathrm{~s}^{-1}$ wide $\mathrm{H} \alpha$ and $\mathrm{H} \beta$ emission after +163 day (phases are corrected for time dilation and are relative to the GROND $r$-band maximum on MJD = 57,924.011). We compare the spectral properties of SN 2017ens to those of other SLSNe and broadlined SNe Ic (SNe Ic-BL), and also present the detections of rarely seen coronal lines. The bolometric light curve and modeling results are described in Section 4. Finally, in Section 5 we discuss plausible scenarios that may explain the spectral evolution and luminosity of SN 2017ens. We adopt a cosmology of $H_{0}=$ $72 \mathrm{~km} \mathrm{~s}^{-1} \mathrm{Mpc}^{-1}, \Omega_{\Lambda}=0.73$, and $\Omega_{m}=0.27$. The foreground reddening toward SN 2017ens is $A_{V}=0.058 \mathrm{mag}$ (Schlafly \& Finkbeiner 2011), and we assume that host-galaxy extinction is negligible because no Na I D absorption is visible in the SN spectrum.

\section{Observations}

Our photometric coverage of SN 2017ens spans the ultraviolet (UV) with the Ultraviolet and Optical Telescope (UVOT) on the Neil Gehrels Swift Observatory, optical wavelengths with GROND, ATLAS, LCO $1 \mathrm{~m},{ }^{34}$ and Lulin-SLT, ${ }^{35}$ and nearinfrared (NIR) bands with GROND. We use standard procedures to reduce the data (Poole et al. 2008 for UVOT; Krühler et al. 2008 for GROND). Ground-based optical photometry is calibrated against the Sloan Digital Sky Survey (SDSS). For ATLAS magnitudes we apply passband corrections using spectra (prescription from Inserra et al. 2018b); for Super Light Telescope (SLT) data we use the conversion of R. Lupton. ${ }^{36}$ The NIR magnitudes are calibrated against Two Micron All Sky Survey (2MASS) field stars. All data are reported in the AB system, and errors include the statistical and systematic uncertainties. We do not have host-galaxy templates, but we estimate a $<15 \%$ contribution from host light $(r>23 \mathrm{mag}$ measured in pre-explosion Panoramic Survey Telescope and Rapid Response System (PanSTARRS) images) to our SN photometry after +150 day. Our photometric results are given in a machine-readable table and shown in Figure 1 (top panel).

We obtained a series of spectra of SN 2017ens, following the SN evolution from +4 day to +265 day (log of observations in Table 1). Spectra are reduced in the standard fashion (ALFOSCGUI pipeline ${ }^{37}$ for ALFOSC) or using custom-built pipelines PyWiFeS (Childress et al. 2014) for WiFeS, LPipe ${ }^{38}$ for LRIS, Krühler et al. (2015) for X-Shooter, and Smartt et al. (2015) for EFOSC2. Finally, we correct the spectral-flux calibration against $r$-band photometry. The resulting calibration error estimated by comparing to $g$-band photometry is generally $<0.10 \mathrm{mag}$, with the exception of the WiFeS $(0.15 \mathrm{mag})$ and Keck $(0.25 \mathrm{mag})$ spectra. (Those data were taken at very high airmass, making flux calibration difficult.) All spectra will be available through WISeREP (Yaron \& Gal-Yam 2012).

\section{Analysis and Results}

\subsection{Light Curves and Comparison}

The discovery epoch of SN 2017ens with $M_{r} \approx-19.8 \mathrm{mag}$ is at MJD $=57,909.3$. ATLAS monitored the field daily for 23 day before discovery. From a deep image taken 3 day before discovery $\left(M_{r} \approx-18.7 \mathrm{mag}\right)$, we constrain the explosion date of SN 2017ens to MJD $=57,907.8 \pm 1.5$; thus, the rest-frame rise time is $\sim 15$ day.

Figure 1 (middle panel) shows the absolute $g$-band light curve, which we compare to SLSNe, SNe IIn, and SNe Ic-BL selected based on the photometric properties and spectral evolution (see Section 3.2) of SN 2017ens. At peak, SN 2017ens is 10 times more luminous than the SNe Ic-BL 1998bw (Patat et al. 2001), 2003jd (Valenti et al. 2008), and SN Ic 2017dio (Kuncarayakti et al. 2018), which shows narrow $\mathrm{H}$ and $\mathrm{He}$ emission in its spectra. The early-phase light-curve evolution of SN 2017ens is

\footnotetext{
${ }^{34}$ https://lco.global/observatory/sites/

$35 \mathrm{http}: / /$ www.lulin.ncu.edu.tw/slt76cm/slt_introdution.htm

$36 \mathrm{http}: / /$ classic.sdss.org/dr4/algorithms/sdssUBVRITransform.html

${ }^{37} \mathrm{http}: / /$ sngroup.oapd.inaf.it/foscgui.html

38 http://www.astro.caltech.edu/ dperley/programs/lpipe.html
} 

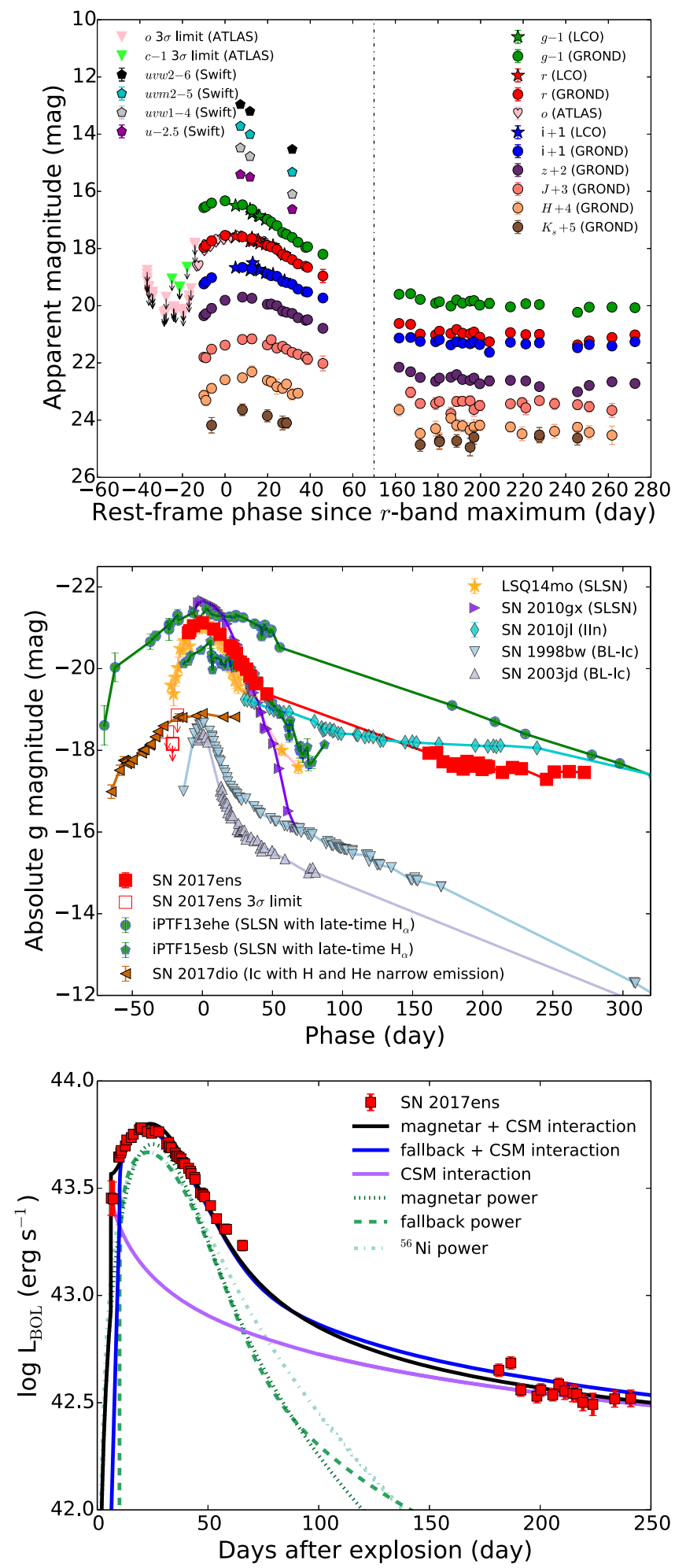

Figure 1. Top panel: multiband light curves of SN 2017ens. Note the discontinuous abscissa. Middle panel: light-curve comparison in absolute $g$-band with the SNe chosen for spectroscopic comparison. Bottom panel: bolometric light curve of SN 2017ens and model fitting. The pseudobolometric luminosity and photometry of SN 2017ens (UV through NIR) is available as data behind the figure. The data used to create this figure are available.

similar to that of rapidly evolving SLSNe such as LSQ14mo (Leloudas et al. 2015; Chen et al. 2017b) and SN 2010gx (Pastorello et al. 2010). SN 2017ens shows no sign of undulations in its light curves, as are often observed in slowly evolving SLSNe as well as SLSNe that exhibit late-time $\mathrm{H} \alpha$ such as iPTF13ehe (Yan et al. 2015) and iPTF15esb (Yan et al. 2017). At late times, the light curves of SN 2017ens remain approximately constant, indicating that strong interaction dominates, as in SN IIn 2010jl before +300 day (Fransson et al. 2014).

\subsection{Spectroscopic Evolution and Comparison}

We show the spectral evolution of SN 2017ens in Figure 2. Around maximum light the spectra are blue and featureless. In the first spectrum taken at +4 day after peak, we detect narrow $\mathrm{H} \alpha$ and $\mathrm{H} \beta$ emission lines (barely resolved width of $\sim 100 \mathrm{~km} \mathrm{~s}^{-1}$ ). Fitting the dereddened spectra with a blackbody gives a temperature of $T_{\mathrm{BB}} \geqslant 10,300 \mathrm{~K}$, consistent with our estimate from the GROND analysis $(\geqslant 11,500 \mathrm{~K})$. At $\sim 1$ month after peak, some broad features emerge, similar to those seen in SNe Ic-BL after peak brightness (e.g., Patat et al. 2001). Apart from narrow $\mathrm{H} \alpha$ and $\mathrm{H} \beta$, we detect a narrow $\mathrm{He} \mathrm{I} \lambda 5876$ emission line. The commonly observed [O II], [O III], and [N II] host-galaxy emission lines are absent, suggesting that the observed Balmer lines originate from the transient itself, not the underlying host (Perley et al. 2017). We also check the WiFeS datacubes and see no [O III] emission at the $\mathrm{SN}$ position.

At late times ( $>160$ day) after the SN emerged from solar conjunction, our data reveal dramatic evolution, with the spectra more resembling those of SNe IIn. The spectra are still blue, but now dominated by prominent, $\sim 2000 \mathrm{~km} \mathrm{~s}^{-1}$ wide Balmer emission lines, indicative of a much stronger interaction with H-rich CSM. The luminosity and the velocity of the $\sim 2000 \mathrm{~km} \mathrm{~s}^{-1} \mathrm{H} \alpha$ line does not vary significantly between +163 and +264 day, staying at $\sim 3 \times 10^{40} \mathrm{erg} \mathrm{s}^{-1}$.

The spectral evolution of $\mathrm{SN} 2017$ ens is unique, sharing features with several distinct $\mathrm{SN}$ subclasses (Figure 3, top panel). In the earliest phases, the blue and featureless spectra share a similarity with young core-collapse SN spectra. We do not see the O II absorption features commonly associated with SLSNe. However, we may have missed them in SN 2017ens. For example, SLSN 2010gx (Pastorello et al. 2010) displayed O II absorption before it peaked and then became blue and featureless.

As the spectra evolve, SN 2017ens is not well matched to other SLSNe such as LSQ14mo (Chen et al. 2017b) and iPTF15esb (Yan et al. 2017). Rather, it appears to be more similar to SNe Ic-BL. The classification tool GELATO (Harutyunyan et al. 2008) applied to the SN 2017ens +27 day spectrum returns the closest similarity with SN 1998bw at +22 day (Patat et al. 2001) and SN 2003jd at -0.3 day (Modjaz et al. 2014). These two SNe Ic-BL still provide a good match to SN 2017ens when we remove the continua assuming a blackbody (Figure 3, middle panel). SN 2017ens has a somewhat bluer continuum, perhaps due to CSM interaction, as was the case for SN 2017dio at +6 day (Kuncarayakti et al. 2018). The origin of the broad feature around $6530 \mathrm{~A}$ is uncertain; it could be attributed to a blend of $\mathrm{Si}$ and $\mathrm{Fe} / \mathrm{Co}$ lines, $\mathrm{H} \alpha$ associated with interaction, or the C II $\lambda 6580$ line sometimes seen in SLSNe (e.g., SN 2018bsz; Anderson et al. 2018).

During the late-time strongly interacting phase, the overall spectral features of SN 2017ens are well matched with those of SN 2017dio at +83 day. Both $\mathrm{SNe}$ exhibit a blue pseudocontinuum (below $\sim 5000 \AA$ ) that is more significant than in 
Table 1

Log of Spectroscopic Observations of SN 2017ens and Its Host Galaxy

\begin{tabular}{|c|c|c|c|c|c|c|c|c|c|}
\hline UT Date & MJD & $\begin{array}{l}\text { Phase } \\
\text { (day) }\end{array}$ & Telescope & Instrument & Grating/Grism /Arm & $\begin{array}{l}\text { Exp. Time } \\
\text { (s) }\end{array}$ & $\begin{array}{l}\text { Slit } \\
\left({ }^{\prime \prime}\right)\end{array}$ & $\begin{array}{l}\text { Resolution } \\
(\AA)\end{array}$ & $\begin{array}{l}\text { Range } \\
(\AA)\end{array}$ \\
\hline 2017 Jun 24 & 57928.392 & 3.95 & ANU $2.3 \mathrm{~m}$ & $\mathrm{WiFeS}^{\mathrm{a}}$ & $\mathrm{B} 3000 / \mathrm{R} 3000$ & $1200 / 1200$ & IFU & $1.6 / 2.5$ & $3500-5700 / 5400-9500$ \\
\hline 2017 Jun 26 & 57930.356 & 5.72 & NOT & ALFOSC & Gr\#4 & 1800 & 1.0 & 16 & 3300-9700 \\
\hline 2017 Jul 20 & 57954.264 & 27.29 & Keck I & LRIS & B600/R400 & 1125 & 0.7 & $5 / 6$ & $3200-10,000$ \\
\hline 2017 Jul 26 & 57960.356 & 32.78 & ANU $2.3 \mathrm{~m}$ & $\mathrm{WiFeS}^{\mathrm{a}}$ & B3000/R3000 & $1200 / 1200$ & IFU & $1.6 / 2.5$ & $3500-5700 / 5400-9500$ \\
\hline 2017 Dec 17 & 58104.325 & 162.65 & NTT & EFOSC2 & Gr\#13 & 2700 & 1.0 & 18.2 & $3700-9200$ \\
\hline 2017 Dec 27 & 58114.301 & 171.65 & NTT & EFOSC2 & $\mathrm{Gr} \# 11 / \mathrm{Gr} \# 16$ & $2700 / 2700$ & $1.0 / 1.0$ & $13.8 / 13.4$ & $3400-7400 / 6000-9900$ \\
\hline 2018 Jan 14 & 58132.275 & 187.86 & VLT & X-Shooter & UVB/VIS/NIR & $3600 / 3400 / 3680$ & $0.9 / 0.9 / 1.0$ & $1 / 1.1 / 3.3$ & $3000-5560 / 5450-10,200 / 10,000-20,600$ \\
\hline 2018 Jan 14 & 58132.312 & 187.90 & NTT & EFOSC2 & Gr\#11 & 3600 & 1.0 & 13.8 & $3400-7400$ \\
\hline 2018 Jan 15 & 58133.263 & 188.75 & VLT & X-Shooter & UVB/VIS/NIR & $7200 / 6800 / 7360$ & $0.9 / 0.9 / 1.0$ & $1 / 1.1 / 3.3$ & $3000-5560 / 5450-10,200 / 10,000-20,600$ \\
\hline 2018 Jan 15 & 58133.273 & 188.76 & NTT & EFOSC2 & $\mathrm{Gr} \# 16$ & 2700 & 1.0 & 13.4 & $6000-9900$ \\
\hline 2018 Jan 16 & 58134.268 & 189.66 & VLT & X-Shooter & UVB/VIS/NIR & $3600 / 3400 / 3680$ & $0.9 / 0.9 / 1.0$ & $1 / 1.1 / 3.3$ & $3000-5560 / 5450-10,200 / 10,000-20,600$ \\
\hline 2018 Jan 19 & 58137.305 & 192.40 & VLT & X-Shooter & UVB/VIS/NIR & $3600 / 3400 / 3680$ & $0.9 / 0.9 / 1.0$ & $1 / 1.1 / 3.3$ & $3000-5560 / 5450-10,200 / 10,000-20,600$ \\
\hline 2018 Feb 14 & 58163.277 & 215.83 & NTT & EFOSC2 & Gr\#11 & 8100 & 1.0 & 13.8 & $3400-7400$ \\
\hline 2018 Feb 18 & 58167.245 & 219.41 & NTT & EFOSC2 & Gr\#16 & 5400 & 1.0 & 13.4 & 6000-9900 \\
\hline 2018 Apr 9 & 58217.065 & 264.35 & NTT & EFOSC2 & Gr\#13 & 2700 & 1.0 & 18.2 & $3650-9200$ \\
\hline
\end{tabular}

Note.

${ }^{a} \mathrm{WiFeS}$ is an integral field unit (IFU) with 25 slitlets that are $1^{\prime \prime}$ wide and $38^{\prime \prime}$ long. Resolution is measured from the night-sky lines. 

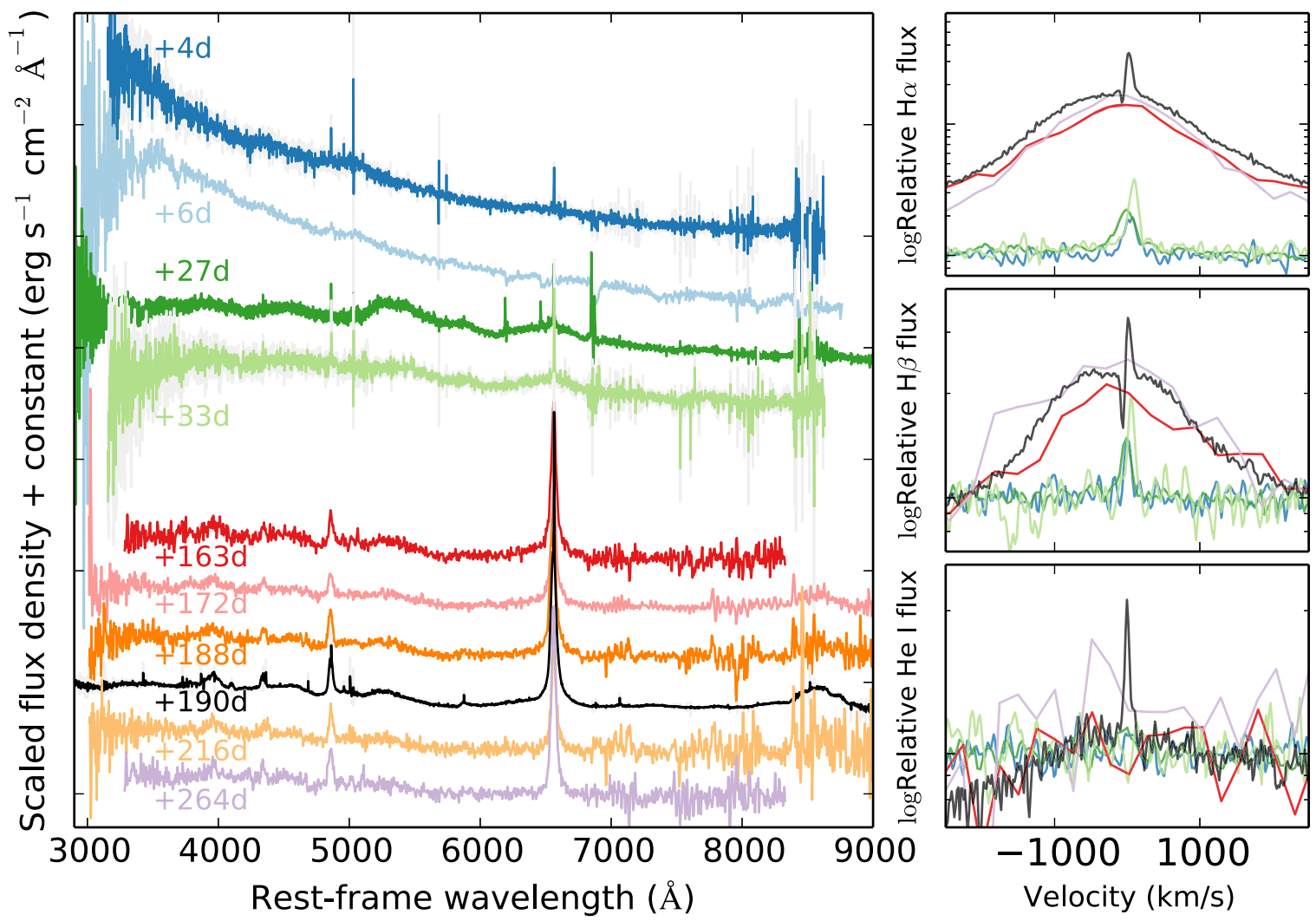

Figure 2. Spectroscopic evolution of SN 2017ens. The right panels show the velocity of the $\mathrm{H} \alpha, \mathrm{H} \beta$, and $\mathrm{He}$ I $\lambda 5876$ lines at selected epochs. Each phase is shown with the same color as in the main panel.

iPTF13ehe at +251 day (Yan et al. 2015); it is likely produced by Fe II lines (Smith et al. 2009).

\subsection{Nebular and Coronal Lines}

The VLT/X-Shooter spectra around +190 day (Figure 4) provide higher resolution and wider wavelength coverage than our other spectra, enabling us to detect many narrow emission lines. Interestingly, we find that the flux ratio of the nebular [O III] $\lambda \lambda 4959,5007$ and auroral [O III] $\lambda 4363$ lines is 0.45 , consistent with coronal lines that may arise from X-ray photoionization (Fransson et al. 2002) of dense gas (see Filippenko \& Halpern 1984, their Figure 11). Therefore, we conclude that the [O III] $\lambda 4363$ line comes from the $\mathrm{SN}$, and we use it to constrain the redshift of SN 2017ens to $z=0.1086$, consistent with the average of the [O II] $\lambda 3727$ and [O III] $\lambda \lambda 4959,5007$ lines.

These narrow coronal lines have been seen in only a handful of SNe IIn and the transitional object SN 2011hw (Pastorello et al. 2015). The ratio $[\mathrm{O} \mathrm{III]}] \lambda 4363 /[\mathrm{O} \mathrm{III}] \lambda \lambda 4959,5007$ for SN 2017ens is similar to that seen in SN 2005ip at +173 day (Smith et al. 2009), SN 2006jd at $+1542 d$ (Stritzinger et al. 2012), and SN 2010jl at +461 day and +573 day (Fransson et al. 2014). Other coronal lines detected in SN 2017ens are similar to those seen in SN 2010jl (Figure 3, bottom panel): [Fe X] $\lambda 6374.5$ is strong, as are [Fe XI] $\lambda 7891.8,[\mathrm{Ne} \mathrm{V}] \lambda \lambda 3345.8,3425.9,[\mathrm{Ca} \mathrm{V}]$ $\lambda 6086.8$, and $[\mathrm{ArX}] \lambda 5533.2$. The presence of these lines is indicative of a highly ionized and dense CSM, although we do not detect the highest-ionization coronal lines such as [Fe XIV] $\lambda 5302.9$ and [Ar XIV] $\lambda 4412.3$, which were seen in SN 2005ip.

The flux ratio of the [O III] $\lambda 4363$ to $\lambda 5007$ lines is a function of the CSM density and temperature. Following Fransson et al. (2014, their Figure 26), we use our measured flux ratio, $\log (\lambda 4363 / \lambda 5007)=-0.22$, to constrain the CSM electron density to lie between $10^{6}$ and $10^{8} \mathrm{~cm}^{-3}$ for $T_{e}=$ 50,000 to $10,000 \mathrm{~K}$. This density range is consistent with that observed for SN 2010jl.

From our mid-resolution X-Shooter data, we resolve narrow P-Cygni profiles on top of the $\sim 2000 \mathrm{~km} \mathrm{~s}^{-1}$ wide Balmer and Paschen lines. We measure the blueshifted wavelength from the absorption component of the $\mathrm{H} \gamma, \mathrm{H} \beta$, and $\mathrm{H} \alpha$ P-Cygni profiles, which suggests that the unshocked CSM has a low velocity of $\sim 50 \mathrm{~km} \mathrm{~s}^{-1}$. A similar velocity of $\sim 60 \mathrm{~km} \mathrm{~s}^{-1}$ is obtained from the P-Cygni profile of the He I $\lambda 10,830$ line. Moreover, we measure the FWHM intensity of the wide components, such as $\mathrm{H} \alpha$ $\left(2500 \pm 700 \mathrm{~km} \mathrm{~s}^{-1}\right), \mathrm{H} \beta\left(2300 \pm 400 \mathrm{~km} \mathrm{~s}^{-1}\right), \operatorname{Pa} \gamma(2000 \pm$ $\left.200 \mathrm{~km} \mathrm{~s}^{-1}\right)$, and He I $\lambda 10,830\left(2200 \pm 200 \mathrm{~km} \mathrm{~s}^{-1}\right)$. We also detect narrow absorption lines from the Balmer series (no clear emission), spanning $\mathrm{H} \epsilon$ to $\mathrm{H} 33$ (3659 $\AA$ ).

In addition, we see emission from the $\mathrm{H}$ II region close to the host-galaxy center (see Figure 4, marked B1), as part of a faint galaxy (SDSS J120409.47-015552.4) with $g=21.92 \pm 0.24 \mathrm{mag}$ $\left(M_{g} \approx-16.5 \mathrm{mag}\right)$. These lines have a slightly different redshift $(z=0.1084)$ than SN 2017ens. In particular, the (noisy) detection of the weak auroral [O III] $\lambda 4363$ line indicates a low host-galaxy metallicity of $\sim 0.04-0.4 Z_{\odot}$ using the direct $T_{\mathrm{e}}$-based method. If 

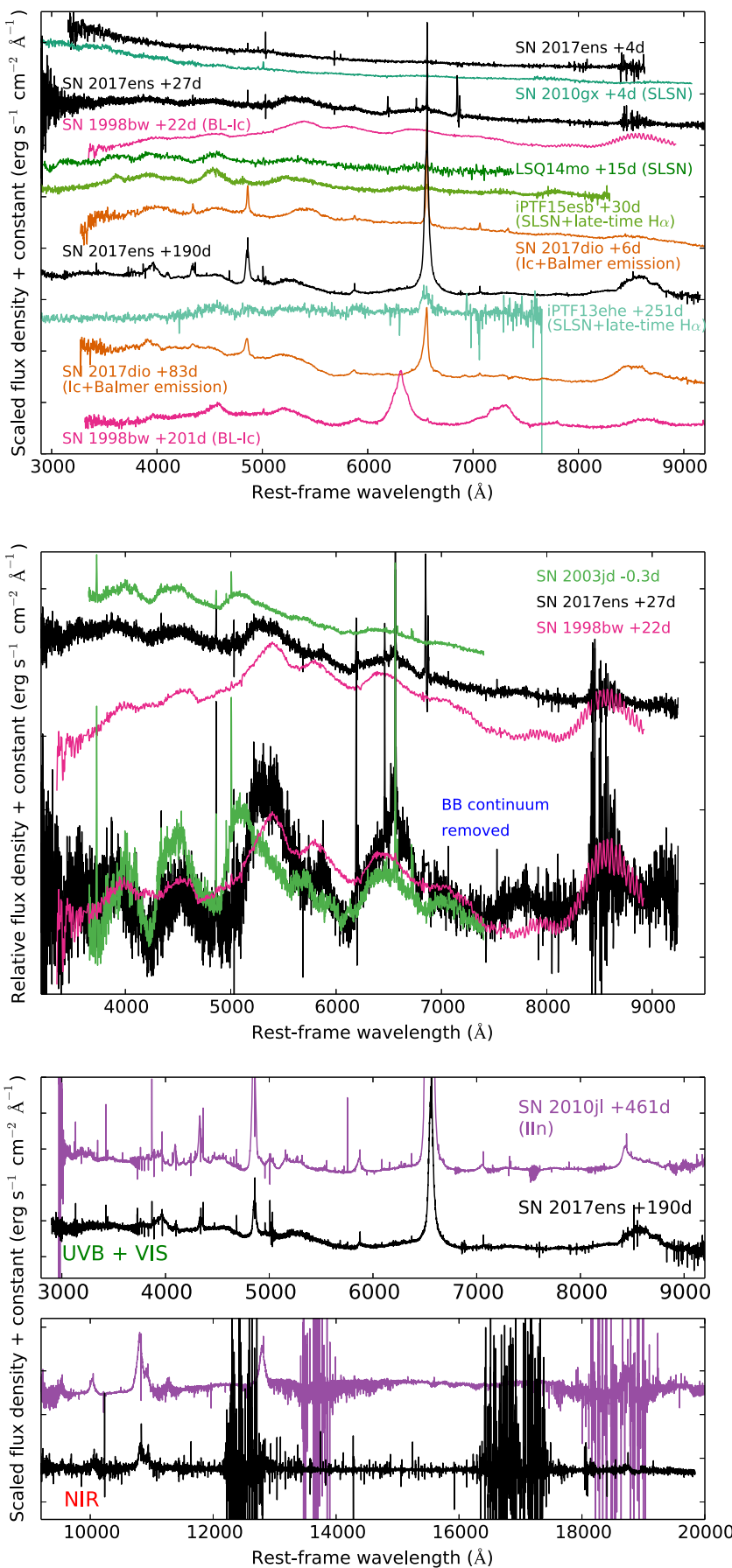

Figure 3. Top panel: comparison between SN 2017ens and other SNe at three selected epochs. Middle panel: comparison between SN 2017ens and the Type Ic-BL SNe 1998bw and 2003jd. The lower spectra have their continuum removed assuming a blackbody. Bottom panel: comparison between SN 2017ens and the Type IIn SN 2010jl at optical and NIR wavelengths.

we instead use the empirical N2 metallicity diagnostic (Pettini \& Pagel 2004), we measure $Z=0.3 \pm 0.2 Z_{\odot}$

\section{Bolometric Light Curve and Model Fitting}

Using all of our available UV-through-NIR photometry, we built a pseudobolometric light curve for SN 2017ens using the prescription from Inserra et al. (2018b). The results are very similar to those derived when using a blackbody fit, as expected as our photometry covers a large wavelength range.
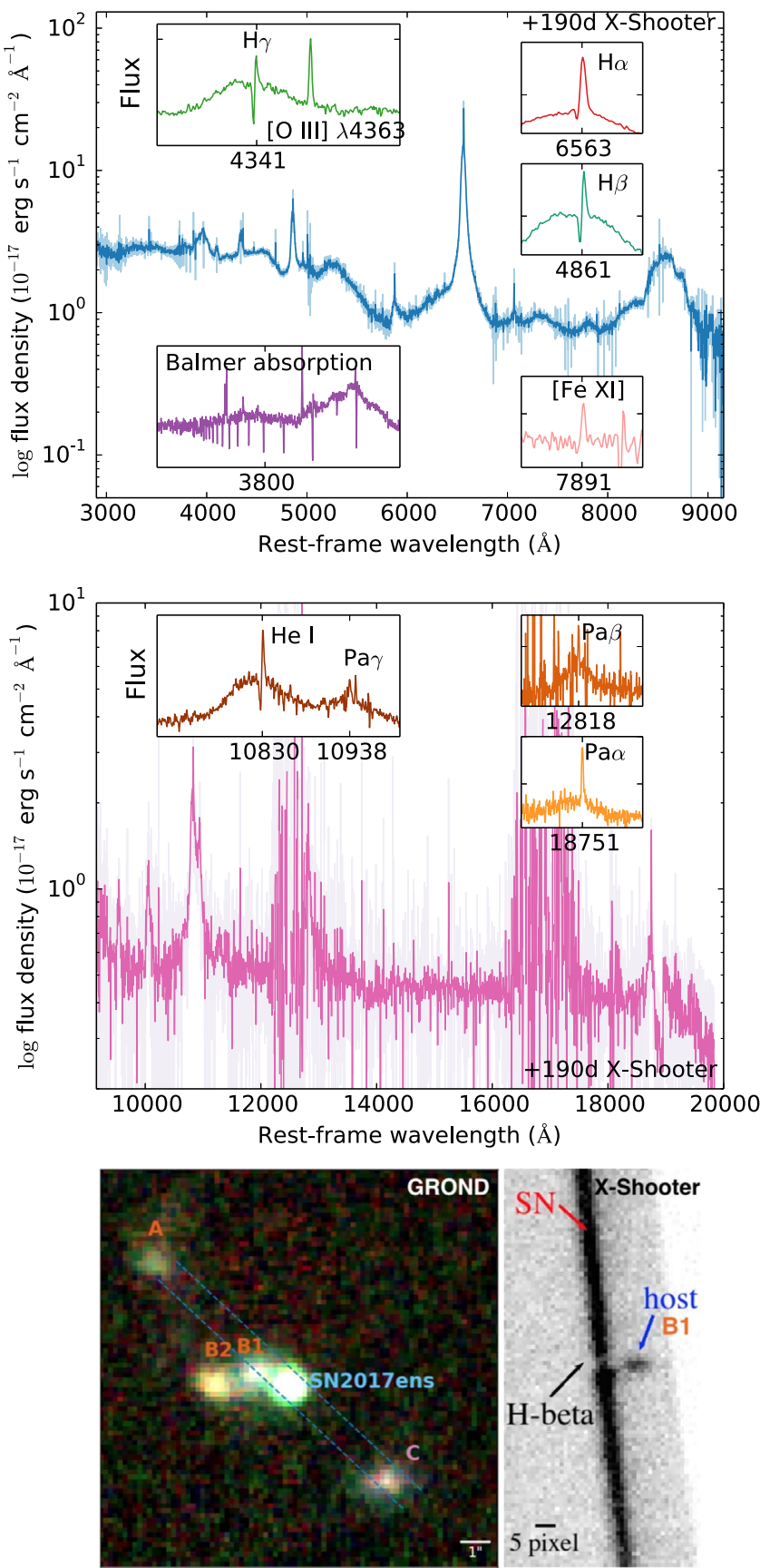

Figure 4. VLT/X-Shooter spectra of SN 2017ens at +190 day. Top panel: UVB and optical (VIS) parts. Middle panel: NIR part. The main panels use a $\log$ scale in order to present details in the continuum (rebinned to $2 \AA$ pixel $^{-1}$ (UVB+VIS) and $5 \AA$ pixel $^{-1}$ (NIR)); inset panels use a linear scale without binning. Bottom panel, left: color-combined image from GROND $r^{\prime} i^{\prime} z^{\prime}$ bands. SN 2017ens is associated with host-galaxy B1+B2, and there is a possible tidal tail connecting to the nearby object A (redshift unknown). Source $\mathrm{C}$ is a background galaxy at $z=0.30$. The X-Shooter slit position is indicated with two dashed lines. Bottom panel, right: the $\mathrm{H} \beta$ position in the two-dimensional raw image.

From a polynomial fit to the bolometric data we obtain $L_{\text {bol }}=(5.86 \pm 0.20) \times 10^{43} \mathrm{erg} \mathrm{s}^{-1}$ at peak and an integrated energy of $(3.53 \pm 1.42) \times 10^{50} \mathrm{erg}$.

To fit our bolometric light curve, we used a two-component model consisting of a central heating and an interaction component. First, the centrally heated component uses the standard Arnett method (Arnett 1982; Inserra et al. 2013). We tested three 
possible central power sources: the nuclear decay of ${ }^{56} \mathrm{Ni}$, the spindown of a magnetar (Kasen \& Bildsten 2010), and fallback accretion (Dexter \& Kasen 2013; Moriya et al. 2018a). The ${ }^{56} \mathrm{Ni}$ decay and the magnetar spindown light curves are obtained as by Inserra et al. (2013), but the magnetar model takes the gamma-ray opacity from the magnetar into account as by Chen et al. (2015). The fallback accretion power is obtained by assuming a central energy input of $L_{\text {fallback, } 1}(t / 1 \mathrm{~s})^{-5 / 3}$, where $L_{\text {fallback, } 1}$ is a constant (Dexter \& Kasen 2013). Second, for the interaction component, we adopted a steady-wind CSM, and the input luminosity from this component goes as $L_{\text {int, } 1}(t / 1 \mathrm{~s})^{-3 / 5}$, where the outer $\mathrm{SN}$ density structure is proportional to $r^{-7}$ (Moriya et al. 2013). The inner SN density structure is assumed to be constant.

We first used the interaction component to fit the bolometric light curve 150 day after explosion, assuming that interaction is the dominant light source at this time. We then derived the contribution required from a central power source at early times to provide a good light-curve match. Given that the spectra of SN 2017ens and SN 1998bw are similar (Figure 3), we used the relation $\left(E_{\mathrm{ej}} / 10^{51} \mathrm{erg}\right) /\left(M_{\mathrm{ej}} / M_{\odot}\right) \approx 3$ found for SN 1998bw (Nakamura et al. 2001) to break the degeneracy between $E_{\mathrm{ej}}$ and $M_{\mathrm{ej}}$.

Figure 1 (bottom panel) shows the results of our fits. In all cases, the CSM interaction model that we used has $L_{\text {int, } 1}=$ $7.7 \times 10^{46} \mathrm{erg} \mathrm{s}^{-1}$. The inner edge of the CSM is set at $1.2 \times 10^{15} \mathrm{~cm}$ to match the early light-curve rise in the model, but this constraint is not strong. We find that all three centrally heated models provide reasonable fits to the bolometric light curve. They all have $E_{\mathrm{ej}}=1.5 \times 10^{52} \mathrm{erg}$ and an ejecta mass of $5 M_{\odot}$. However, the ${ }^{56} \mathrm{Ni}$-powered light curve requires a very high ${ }^{56} \mathrm{Ni}$ mass of $3.5 \mathrm{M}_{\odot}$. This is close to the ejecta mass, and we therefore find the ${ }^{56} \mathrm{Ni}$-powered model to be unlikely. Alternatively, a magnetar central engine with an initial spin of $3.8 \mathrm{~ms}$ and a magnetic field of $8 \times 10^{13} \mathrm{G}$, and fallback accretion with $L_{\text {fallback, } 1}=6 \times 10^{53} \mathrm{erg} \mathrm{s}^{-1}$, provide good qualitative fits to the light curve. It is of course possible that the entire light curve is driven by different degrees of interaction. The contribution of the interaction component at early times (0-70 day after explosion) is $\sim 20 \%$, while it is $\gtrsim 90 \%$ at late times (200 day).

Assuming the above best-fit results and a kinetic energy to radiation conversion efficiency at the shock of 0.1 (Moriya et al. 2013), we estimate the mass-loss rate of the progenitor to be $5 \times 10^{-4} M_{\odot} \mathrm{yr}^{-1}$, with a constant wind velocity of $50 \mathrm{~km} \mathrm{~s}^{-1}$. The CSM density estimate is similar to those of SNe IIn showing similar coronal lines (Taddia et al. 2013).

\section{Discussion}

One important clue to interpreting the possible powering mechanisms behind SN 2017ens is that we measured the $\mathrm{H}$-rich material to have a velocity of $\sim 50-60 \mathrm{~km} \mathrm{~s}^{-1}$ from the blueshifted absorption of the narrow P-Cygni profiles. This wind velocity is far slower than those present in Wolf-Rayet star winds. If this wind is from the progenitor, it could come from a massive H-rich progenitor (such as a luminous blue variable) that explosively ejected its $\mathrm{H}$ envelope shortly before the SN explosion. Alternatively, this wind could come from a pulsational pair-instability $\mathrm{SN}$ with a slow and long-term stable wind (Woosley 2017).

It is also possible that SN 2017ens exploded as a SN Ic-BL inside a patchy, H-rich CSM from a binary companion; the expanding ejecta interact with the bulk of the CSM at later times, as has been suggested for SN 2017dio (Kuncarayakti et al. 2018). Alternatively, as proposed for ASASSN-15no
(Benetti et al. 2018), a dense inner CSM may have hidden the $\mathrm{SN}$ features at early times, before they become briefly visible as the CSM was swept up by the ejecta. At late times they could have again been masked by an increasingly strong interaction component. A special CSM geometry (e.g., doughnut shape) is also probable, and we see the SN Ic-BL along a certain viewing angle.

In the case of a binary companion, the wind of $\sim 50-60 \mathrm{~km} \mathrm{~s}^{-1}$ and mass-loss rate of $5 \times 10^{-4} M_{\odot} \mathrm{yr}^{-1}$ are consistent with a red supergiant (Goldman et al. 2017), albeit at the more extreme end, which can be explained by the companion having gained mass from the SN progenitor during an earlier accretion phase. If so, this may suggest that the progenitor of SN 2017ens lost its $\mathrm{H}$ and He layers through interaction with a binary companion.

We must also consider the apparent $\sim 2000 \mathrm{~km} \mathrm{~s}^{-1}$ material, given its high luminosity. If this is associated with mass loss from the progenitor, and the line width is not from electron scattering as seen in many SNe IIn, then the material is moving much faster than the winds of H-rich stars (or the CSM of SNe IIn). It is difficult to imagine how this could be produced by anything other than a sudden ejection of the $\mathrm{H}$ envelope, shortly before the $\mathrm{SN}$ explosion. In fact, the luminosity of the $\sim 2000 \mathrm{~km} \mathrm{~s}^{-1}$ wide component of $\mathrm{H} \alpha$ is comparable to that seen in $\mathrm{SN} 1995 \mathrm{~N}$ (Fransson et al. 2002) $\left(\sim 2.3 \times 10^{40} \mathrm{erg} \mathrm{s}^{-1}\right)$, and it may be too large to be coming solely from swept-up material. A pulsational pair-instability explosion is at least qualitatively consistent with an outburst that can unbind the $\mathrm{H}$ envelope shortly before an $\mathrm{SN}$ explosion. This scenario is also consistent with the measured low-metallicity environment.

The unique spectroscopic evolution of SN 2017ens together with its high luminosity poses challenges to all currently known SN scenarios. While detailed modeling can help elucidate the nature of this transient, ongoing surveys for SLSNe such as GREAT will find more such peculiar transients. With a larger sample and high-cadence follow-up spectroscopy, we will be able to further understand the nature of SN 2017enslike objects and the role of interaction in SLSNe.

T.W.C. acknowledges Thomas Krühler for the X-Shooter data reduction, Lin Yan and Claes Fransson for providing comparison spectra, Jason Spyromilio for useful discussions, Chien-Hsiu Lee and You-Hua Chu for coordinating observational resources, and funding from the Alexander von Humboldt Foundation. M.F. acknowledges the support of a Royal Society-Science Foundation Ireland University Research Fellowship. P.S. acknowledges support through the Sofia Kovalevskaja Award (Alexander von Humboldt Foundation). A.V.F. is grateful for the support of the TABASGO Foundation, the Christopher R. Redlich fund, and the Miller Institute for Basic Research in Science (U.C. Berkeley). A.J.R. and I.R.S. are supported by the Australian Research Council through grants FT170100243 and FT160100028, respectively. F.T. and J.S. acknowledge support from the KAW Foundation. S.J.S. acknowledges funding from the European Research Council Grant agreement \#291222 and STFC grant ST/ P000312/1. M.G. is supported by Polish National Science Centre grant OPUS 2015/17/B/ST9/03167. K.M. acknowledges support from the UK STFC through an Ernest Rutherford Fellowship and from a Horizon 2020 ERC Starting Grant (\#758638). L.G. was supported in part by US NSF grant AST1311862. C.P.G. acknowledges support from EU/FP7-ERC 
grant \#615929. Z.Y.L., C.C.N., and P.C.Y. are grateful for funding from MoST (Taiwan) under grants 105-2112-M-008002-MY3, 104-2923-M-008-004-MY5, and 106-2112-M-008007. A.P. and S.B. are partially supported by PRIN-INAF 2017 "Toward the SKA and CTA era: discovery, localization, and physics of transient sources" (P.I.: Giroletti). A.G.-Y. is supported by the EU via ERC grant No. 725161, the Quantum Universe I-Core program, the ISF, the BSF Transformative program, and a Kimmel award.

Part of the funding for GROND was generously granted from the Leibniz Prize to Prof. G. Hasinger (DFG grant HA 1850/28-1). Some observations were made with the Nordic Optical Telescope using ALFOSC. This publication has made use of data collected at Lulin Observatory, partly supported by MoST grant 105-2112-M-008-024-MY3. Some of the data presented herein were obtained at the W. M. Keck Observatory, which is operated as a scientific partnership among the California Institute of Technology, the University of California, and NASA; the observatory was made possible by the generous financial support of the W. M. Keck Foundation.

\section{ORCID iDs}

T.-W. Chen (1) https://orcid.org/0000-0003-1532-0149

C. Inserra (ㄴ) https://orcid.org/0000-0002-3968-4409

M. Fraser (ㄷ https://orcid.org/0000-0003-2191-1674

T. J. Moriya (1) https://orcid.org/0000-0003-1169-1954

A. V. Filippenko (1) https://orcid.org/0000-0003-3460-0103

D. A. Perley (1) https://orcid.org/0000-0001-8472-1996

A. J. Ruiter (i) https://orcid.org/0000-0002-4794-6835

I. Seitenzahl iㅏ https://orcid.org/0000-0002-5044-2988

J. Sollerman (10) https://orcid.org/0000-0003-1546-6615

J. P. Anderson (1) https://orcid.org/0000-0003-0227-3451

A. Jerkstrand (i) https://orcid.org/0000-0001-8005-4030

C.-C. Ngeow (1) https://orcid.org/0000-0001-8771-7554

S. J. Smartt (1) https://orcid.org/0000-0002-8229-1731

S. Taubenberger (1) https://orcid.org/0000-0002-4265-1958

M. Berton (1) https://orcid.org/0000-0002-1058-9109

M. Della Valle (i) https://orcid.org/0000-0003-3142-5020

L. Galbany (i) https://orcid.org/0000-0002-1296-6887

A. Gal-Yam (i) https://orcid.org/0000-0002-3653-5598

A. Heinze (1) https://orcid.org/0000-0003-3313-4921

H. Kuncarayakti (i) https://orcid.org/0000-0002-1132-1366

G. Leloudas (1) https://orcid.org/0000-0002-8597-0756

P. Mazzali (1) https://orcid.org/0000-0001-6876-8284

A. Rau (ib https://orcid.org/0000-0001-5990-6243

B. Stalder (i) https://orcid.org/0000-0003-0973-4900

P.-C. Yu (1) https://orcid.org/0000-0001-8894-0854

\section{References}

Anderson, J. P., Pessi, P. J., Dessart, L., et al. 2018, arXiv:1806.10609 Arnett, W. D. 1982, ApJ, 253, 785

Benetti, S., Zampieri, L., Pastorello, A., et al. 2018, MNRAS, 476, 261 Chen, T.-W., Inserra, C., Smartt, S. J., et al. 2017a, ATel, 10478, 1

Chen, T.-W., Nicholl, M., Smartt, S. J., et al. 2017b, A\&A, 602, A9

Chen, T.-W., Schady, P., Kruehler, T., et al. 2017c, ATel, 10510, 1

Chen, T.-W., Smartt, S. J., Jerkstrand, A., et al. 2015, MNRAS, 452, 1567

Chevalier, R. A., \& Irwin, C. M. 2011, ApJL, 729, L6

Childress, M. J., Vogt, F. P. A., Nielsen, J., \& Sharp, R. G. 2014, Ap\&SS, 349,617

Dexter, J., \& Kasen, D. 2013, ApJ, 772, 30

Filippenko, A. V. 1997, ARA\&A, 35, 309

Filippenko, A. V., \& Halpern, J. P. 1984, ApJ, 285, 458

Fransson, C., Chevalier, R. A., Filippenko, A. V., et al. 2002, ApJ, 572, 350

Fransson, C., Ergon, M., Challis, P. J., et al. 2014, ApJ, 797, 118

Gal-Yam, A. 2012, Sci, 337, 927

Gal-Yam, A. 2017, in Observational and Physical Classification of Supernovae, ed. A. W. Alsabti \& P. Murdin (Dordrecht: Springer), 195

Goldman, S. R., van Loon, J. T., Zijlstra, A. A., et al. 2017, MNRAS, 465, 403 Greiner, J., Bornemann, W., Clemens, C., et al. 2008, PASP, 120, 405

Harutyunyan, A. H., Pfahler, P., Pastorello, A., et al. 2008, A\&A, 488, 383 Inserra, C., Prajs, S., Gutierrez, C. P., et al. 2018a, ApJ, 854, 175

Inserra, C., Smartt, S. J., Gall, E. E. E., et al. 2018b, MNRAS, 475, 1046

Inserra, C., Smartt, S. J., Jerkstrand, A., et al. 2013, ApJ, 770, 128

Kasen, D., \& Bildsten, L. 2010, ApJ, 717, 245

Krühler, T., Küpcü Yoldaş, A., Greiner, J., et al. 2008, ApJ, 685, 376

Krühler, T., Malesani, D., Fynbo, J. P. U., et al. 2015, A\&A, 581, A125

Kuncarayakti, H., Maeda, K., Ashall, C. J., et al. 2018, ApJL, 854, L14

Leloudas, G., Patat, F., Maund, J. R., et al. 2015, ApJL, 815, L10

Mauerhan, J. C., Filippenko, A. V., Zheng, W., et al. 2018, arXiv:1803.07051

Milisavljevic, D., Margutti, R., Kamble, A., et al. 2015, ApJ, 815, 120

Modjaz, M., Blondin, S., Kirshner, R. P., et al. 2014, AJ, 147, 99

Moriya, T. J., Maeda, K., Taddia, F., et al. 2013, MNRAS, 435, 1520

Moriya, T. J., Nicholl, M., \& Guillochon, J. 2018a, arXiv:1806.00090

Moriya, T. J., Sorokina, E. I., \& Chevalier, R. A. 2018b, SSRv, 214, 59

Nakamura, T., Mazzali, P. A., Nomoto, K., \& Iwamoto, K. 2001, ApJ, 550, 991

Pastorello, A., Benetti, S., Brown, P. J., et al. 2015, MNRAS, 449, 1921

Pastorello, A., Smartt, S. J., Botticella, M. T., et al. 2010, ApJL, 724, L16

Patat, F., Cappellaro, E., Danziger, J., et al. 2001, ApJ, 555, 900

Perley, D. A., Chen, T.-W., Filippenko, A. V., Brink, T. G., \& Zheng, W. 2017, ATel, 10587, 1

Pettini, M., \& Pagel, B. E. J. 2004, MNRAS, 348, L59

Poole, T. S., Breeveld, A. A., Page, M. J., et al. 2008, MNRAS, 383, 627

Quimby, R. M., Kulkarni, S. R., Kasliwal, M. M., et al. 2011, Natur, 474, 487

Schlafly, E. F., \& Finkbeiner, D. P. 2011, ApJ, 737, 103

Smartt, S. J., Valenti, S., Fraser, M., et al. 2015, A\&A, 579, A40

Smith, N., Silverman, J. M., Chornock, R., et al. 2009, ApJ, 695, 1334

Stritzinger, M., Taddia, F., Fransson, C., et al. 2012, ApJ, 756, 173

Taddia, F., Stritzinger, M. D., Sollerman, J., et al. 2013, A\&A, 555, A10

Tonry, J. L., Denneau, L., Heinze, A. N., et al. 2018, PASP, 130, 064505

Valenti, S., Benetti, S., Cappellaro, E., et al. 2008, MNRAS, 383, 1485

Woosley, S. E. 2010, ApJL, 719, L204

Woosley, S. E. 2017, ApJ, 836, 244

Yan, L., Lunnan, R., Perley, D. A., et al. 2017, ApJ, 848, 6

Yan, L., Quimby, R., Ofek, E., et al. 2015, ApJ, 814, 108

Yaron, O., \& Gal-Yam, A. 2012, PASP, 124, 668 